\title{
New Adipocytokines
}

\section{Mansour SW*}

Department of Biomedical Sciences, King Faisal University, Saudi Arabia

*Corresponding author: Mansour SW, Professor, Department of Biomedical Sciences, King Faisal University, Saudi Arabia; Tel: 5800000-1801-237, E-mail: drsherif36@hotmail.com

Citation: Mansour SW (2013) New Adipocytokines. J Pharm Drug Devel 1(1): e103. doi: 10.15744/23489782.1.e103

Received Date: June 18, 2013 Accepted Date: July 29, 2013 Published Date: August 01, 2013

They are cytokines secreted by adipose tissue considered to be immunomodulating agents, however they can be more accurately put into the larger, growing list of adipose-derived hormones.

It includes:

1. Chemerin

2. Interleukin-6 (IL-6)

3. Monocyte chemotactic protein-1 (MCP-1)

4. Plasminogen activator inhibitor-1 (PAI-1)

5. Retinol binding protein 4 (RBP4)

6. Tumor necrosis factor - Alpha (TNFa)

7. Visfatin

8. Leptin

9. Adiponectin

10. Apelin

\section{Chemerin, also Known as Retinoic Acid Re- ceptor Responder Protein 2 (RARRES2)}

In humans, chemerin mRNA is highly expressed in white adipose tissue, liver and lung while its receptor, CMKLR1 is predominantly expressed in immune cells as well as adipose tissue. Because of its role in adipocyte differentiation and glucose uptake, chemerin is classified as an adipokine.

\section{Effects as an adipocytokine}

Chemerin has been implicated in autocrine / paracrine signaling for adipocyte differentiation and also stimulation of lipolysis. Genetic knockdown of chemerin or its receptor, CMKLR1 impairs differentiation into adipocytes, and reduces the expression of GLUT4 and adiponectin, while increasing expression of IL-6 and insulin receptor. Furthermore, postdifferentiation knockdown of chemerin reduced GLUT4, leptin, adiponectin,perilipin, and reduced lipolysis, suggesting chemerin plays a role in metabolic function of mature adipocytes.

Studies in mice have shown neither chemerin nor CMKLR1 are highly expressed in brown adipose tissue, indicating that chemerin plays a role in energy storage rather than thermogenesis.

\section{Effects in obesity and diabetes}

Given chemerin's role as a chemoattractant and a recent finding macrophages have been implicated in chronic inflammation of adipose tissue in obesity. This suggests chemerin may play an important in the pathogenesis of obesity and insulin resistance.

\section{Nicotinamide Phosphoribosyltransferase also Known as Visfatin is an Enzyme that in $\mathrm{Hu}-$ mans}

The protein is an adipokine that is localized to the bloodstream and has various functions, including the promotion of vascular smooth muscle cell maturation and inhibition of neutrophil apoptosis. It also activates insulin receptor and has insulin-mimetic effects, lowering blood glucose and improving insulin sensitivity. The protein is highly expressed in visceral fat and serum levels of the protein correlate with obesity.

\section{Apelin}

Apelin is a new peptide that was identified in 1998.

\section{Vascular effects}

Vascular expression of the receptor participates in the control of blood pressure and its activation promotes the formation of new blood vessels (angiogenesis). The hypotensive effect of apelin results from the activation of receptors expressed at the surface of endothelial cells. This activation induces the release of NO, a potent vasodilator, which induces relaxation of the smooth muscle cells of artery wall. The angiogenic activity is the consequence of apelin action on the proliferation and migration of the endothelial cells. 


\section{Cardiac effects}

Its expression is detected in the cardiomyocytes of the adult where apelin behaves as one of the most potent stimulator of cardiac contractility. It is one of the most potent stimulators of cardiac contractility yet identified, and plays a role in cardiac tissue remodeling. In addition, apelin regulates fluid homeostasis. It is important in the hypothalamic regulation of food and water intake, and pituitary hormone release.

\section{Brain effects}

Apelin receptor is involved in water and food intake. Apelin injection increases water intake and apelin decreases in the hypothalamus the secretion of $\mathrm{ADH}$, antidiuretic hormone, vasopressin.

\section{Digestive effects}

In stomach, activation of receptors on enterochromaffine-like cells by apelin secreted by parietal cells can inhibit histamine release by enterochromaffine-like cells, which in turn decreases acid secretion by parietal cells. In pancreas, apelin inhibits the insulin secretion induced by glucose.

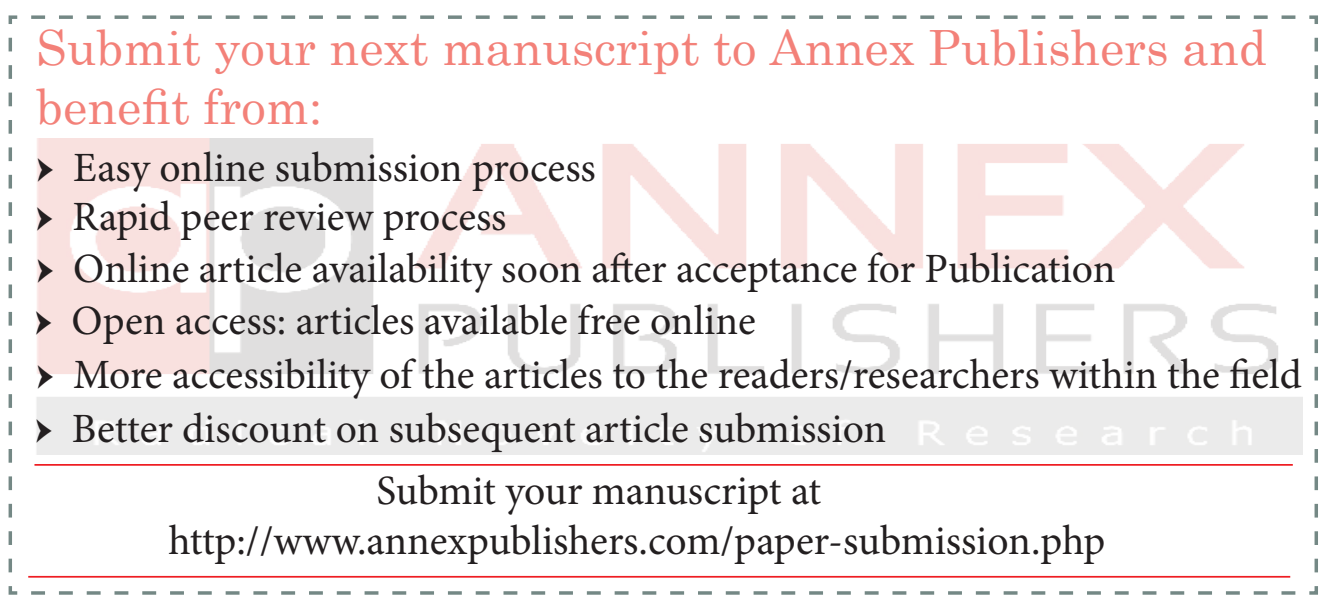

